\title{
Impact of Pharmacovigilance in Healthcare System: Regulatory Perspective
}

\section{Suryakanta Swain* and Chinam Niranjan Patra}

Department of Pharmaceutics, Roland Institute of Pharmaceutical Sciences, Berhampur-760 010, Odisha, India

\section{Importance of Open Access and Special Features of Omics Group}

Open access plays a pivotal role in propagating scientific information globally. It is completely user friendly and is open for all to access easily. Its salient features are, that it covers 50 world's leading languages and have the facility of audio versions too. It invites every individual to share as well as explore scientific knowledge and research in the field of pharmaceutical science. As it is similar to social networking it meets the requirements of young generation and provides an excellent platform for new pharma scholars. Share important information about innovative technologies and other regulatory affairs. It enhances scientific skills of the authors, readers, reviewers, editors and advisors by providing scientific credits. On whole it encourages each and everyone and attracts all towards the majestic world of science.

\section{Structure of the Editorial}

The World Health Organization (WHO) had defined pharmacovigilance (PV) as "the science and activities relating to the detection, assessment, understanding, and prevention of adverse effects or any other possible drug-related problems. The PV system safeguards the public through efficient and timely identification, collection, and assessment of medicine-related adverse events and by communicating risks and benefits to support decision making about medicines at various levels of the health care system. The pharmacovigilance (PV) system safeguards the public through efficient and timely identification, collection, assessment, and communication of medicine-related adverse events. A comprehensive PV system includes both active and passive surveillance methods, effective mechanisms to communicate medicine safety information to health care professionals and the public, collaboration among a wide range of partners and organizations, and incorporation of PV activities into the various levels of the health system, from the facility to the national levels. PV systems should include all entities and resources that protect the public from medicines-related harm, whether in personal health care or public health services. The capacity to conduct medicine safety research exists in Africa, yet active approaches to identify and evaluate medicine-related risks are limited. Adverse drug events (ADEs) from poor product quality, adverse drug reactions (ADRs), and medication errors contribute significantly to morbidity and mortality. Besides the economic consequences, cases of adverse events affect the credibility of the health system leading to loss of confidence. According to a 2011 assessment of pharmacovigilance system by management sciences by health (MSH), it is estimated that the sub-saharan Africa has an estimated pharmaceutical market size of 3.8 billion to 4.7 billion US dollars (USD) and local manufacturing capacity in 80 percent of countries, capacity for regulating health products in SSA is inadequate. Currently, 74 percent of these 46 countries have a national medicines regulatory authority (NMRA), 78 percent have a national medicine policy (NMP), 5 World Health Organization (WHO) prequalified quality control laboratories exist in the region, and 33 SSA countries are an official or associate member of the WHO Programme for International Drug Monitoring. The report also highlighted that Most of the SSA countries did not have procedures for managing or minimizing important known harmful effects of highrisk medicines. There was no formal risk management activity designed to prevent or minimize problems related to the medicine. The study found risk management and communication as the PV component with the weakest system and performance.

\section{Pharmacovigilance and International Health}

The current global network of pharmacovigilance centers is coordinated by the Uppsala Monitoring Centre, would be strengthened by an independent system of review. This would consider contentious and important drug safety issues that have the potential to affect public health adversely beyond national boundaries. The Erice Declaration provides a framework of values and practice for collection, analysis and subsequent communication of drug safety issues. Today, the burden of ADRs on public health despite the progress in pharmacovigilance that has been made, the burden on public health of ADRs remains significant. Drug utilization patterns are a major determinant in drug safety. For instance, the use of injectable medicines is more common in developing countries. Direct advertizing to the consumer of prescription medicines has become commonplace in many countries. With this information patients feel more able to make their own therapeutic decisions, without assistance from doctor or pharmacist. The result has been increasing self-medication, licit and illicit sale of medicines over the Internet, and over-prescribing by doctors on patients' demand. This has had considerable effect on increased prescribing. Such public health programmes, however, need not focus only on patients but could be used for the benefit of the general public as well. Such awareness-building and educational initiatives should also include children and elderly populations and could be greatly facilitated through partnerships with the media, educational institutions, governmental and non-governmental organizations. The success of WHO International Drug Monitoring Programmes is entirely dependent on the contributions of national pharmacovigilance centers. Ideally every country should have a pharmacovigilance centre.

\section{Pharmacovigilance in Drug Regulation}

Pharmacovigilance programs made strong by links with regulators and the regulators understand that pharmacovigilance plays a pivotal role in ensuring ongoing safety of medicine. From the current status it has been reported that substantial increase in the number of clinical trials in developed and developing countries and soon after the approval of clinical trials, regulatory bodies look at safety and efficacy of new drug products under investigation as safety monitoring of

*Corresponding author: Suryakanta Swain, Roland Institute of Pharmaceutical Sciences, Department of Pharmaceutics, Roland Institute of Pharmaceutical Sciences, Berhampur-760 010, Odisha, India, Tel: +91-9438038643; Fax: +91680-2404112; E-mail: swain_suryakant@yahoo.co.in

Received November 07, 2014; Accepted November 08, 2014; Published November 10, 2014

Citation: Swain S, Patra CN (2014) Impact of Pharmacovigilance in Healthcare System: Regulatory Perspective. Pharmaceut Reg Affairs 3: e143. doi:10.4172/2167-7689.1000e143

Copyright: $\odot 2014$ Swain S, et al. This is an open-access article distributed under the terms of the Creative Commons Attribution License, which permits unrestricted use, distribution, and reproduction in any medium, provided the original author and source are credited. 
medicines is an integral part of clinical practice. Education and training of health professionals in medicine safety, exchange of information between national pharmacovigilance centers, the coordination of such exchange, and the linking of clinical experience of medicine safety with research and health policy, all serve to enhance effective patient care. A regular flow and exchange of information in this way means that national pharmacovigilance programmes are ideally placed to identify gaps in our understanding of medicine-induced diseases. The Council for International Organizations of Medical Sciences (CIOMS) report on benefit-risk assessment of medicines after marketing has contributed to a more systematic approach to determining the merit of available medicines. The provision of good quality, safe and effective medicines and their appropriate use is the responsibility of national governments. Multidisciplinary collaboration is of great importance in particular, links need to be forged between various departments of the ministry of health and also with other stakeholders, such as the pharmaceutical industry, universities, nongovernmental organizations (NGOs) and those professional associations having responsibility for education on rational use of medicines and pharmacotherapy monitoring.

\section{Pharmacovigilance in Disease Control Public Health Programmes}

The monitoring of medicine safety in countries where there is no regulatory or safety monitoring system in place, or in remote areas with little or no health care surveillance or infrastructure, has been identified as a matter for concern. The problems are especially apparent in situations that involve the use of medicines in specific communities, for example, for the treatment of tropical diseases such as malaria, leishmaniasis and schistosomiasis, and for the treatment of HIV/AIDS and tuberculosis.

\section{Worldwide Soldiers of Pharmacovigilance}

A complex and vital relationship exists between wide ranges of partners in the practice of drug safety monitoring. These partners must jointly anticipate, understand and respond to the continually increasing demands and expectations of the public, health administrators, policy officials, politicians and health professionals. The team is a part of the Department of Essential Drugs and Medicines Policy, within the WHO Health Technology and Pharmaceuticals cluster. The purpose of the department is to save lives and improve health by closing the huge gap between the potential that essential drugs have to offer and the reality that for millions of people, particularly the poor and disadvantaged, medicines are unavailable, unaffordable, unsafe or improperly used. The entire cost of a pharmacovigilance system, compared with the national expenditure on medicines or the cost of ADRs to the nation is very small indeed. A number of medical institutions have developed adverse reaction and medication error close watch systems in their clinics, wards and emergency rooms. Case-control studies and other pharmacoepidemiological methods have increasingly been used to estimate the harm associated with medicines once they have been marketed. Direct patient participation in the reporting of drug related problems will increase the efficiency of the pharmacovigilance system and compensate for some of the shortcomings of systems based on reports from health professionals only.

On behalf of the editor and editorial board member for this journal, I would like to thanks the OMICS publishing group 5716 Corsa Ave, Suite 110 Westlake, Los Angeles, A 91362-7354, USA for giving me another opportunity for writing an invited editorial article for publication in the upcoming issue. 